\title{
Drosophila blood as a model system for stress sensing mechanisms
}

\author{
Jiwon Shim ${ }^{*}$ \\ Department of Life Science, College of Natural Science, Hanyang University, Seoul 133-791, Korea
}

\begin{abstract}
The Drosophila lymph gland is the hematopoietic organ in which stem-like progenitors proliferate and give rise to myeloid-type blood cells. Mechanisms involved in Drosophila hematopoiesis are well established and known to be conserved in the vertebrate system. Recent studies in Drosophila lymph gland have provided novel insights into how external and internal stresses integrate into blood progenitor maintenance mechanisms and the control of blood cell fate decision. In this review, I will introduce a developmental overview of the Drosophila hematopoietic system, and recent understandings of how the system uses developmental signals not only for hematopoiesis but also as sensors for stress and environmental changes to elicit necessary blood responses. [BMB Reports 2015; 48(4): 223-228]
\end{abstract}

\section{DROSOPHILA LYMPH GLAND AS A HEMATOPOIETIC MODEL SYSTEM}

Drosophila hematopoiesis is divided largely into two waves and an additional wave: embryonic, lymph gland, and larval hematopoiesis (1). The initial wave of hematopoiesis takes place in the embryonic head mesoderm, where undifferentiated blood cells are formed, proliferate, and differentiate into mature blood cell types, including plasmatocytes and crystal cells (2). Upon differentiation, embryonic blood cells disperse through the embryo and migrate throughout along specific paths (3). Later in the larval stage, an independent set of blood cells are found in the lymph gland, which originate from the cardiogenic mesoderm (4-6). The lymph gland is composed of three compartments. Stem-like progenitors in the lymph glands locate in the core, called the Medullary zone (MZ), and the $M Z$ cells give rise to mature blood cells in the outermost region,

*Corresponding author. Tel: +82-2-2220-2615; Fax: +82-2-22980319; E-mail: jshim@hanyang.ac.kr

http://dx.doi.org/10.5483/BMBRep.2015.48.4.273

\section{Received 7 October 2014}

Keywords: Adenosine, Blood progenitors, Drosophila, Hypoxia, Insulin, Lymph gland, Olfaction, Progenitor, ROS, Starvation, Stem cell, Stress called the Cortical zone (CZ) (Fig. 1) (6). Similar to other stem cell systems, maintenance of the stem-like progenitors is controlled mainly by a microenvironment niche, also known as the Posterior signaling center (PSC) $(7,8)$. Differentiation of the stem-like progenitors produces at least three types of blood cells that are reminiscent of vertebrate myeloid cell lineages (6). Plasmatocytes, corresponding to vertebrate macrophages, represent more than $95 \%$ of total blood cells and function in cellular immunity and phagocytosis $(2,4,5)$. Crystal cells are named after their resident crystalline structures of unidentified proteins and are known to mediate wound healing and melanization, similar to platelet in vertebrates $(9,10)$. While crystal cells represent approximately $5 \%$ of total blood cells under normal conditions, there is a specific cell type, called lamellocytes, that only appears upon immune challenge and takes part in the encapsulation of relatively large particles $(11,12)$.

The embryonic phase of hematopoiesis corresponds to the 'primitive' hematopoiesis of vertebrates and the lymph gland phase of hematopoiesis is referred to as the 'definitive' hema-

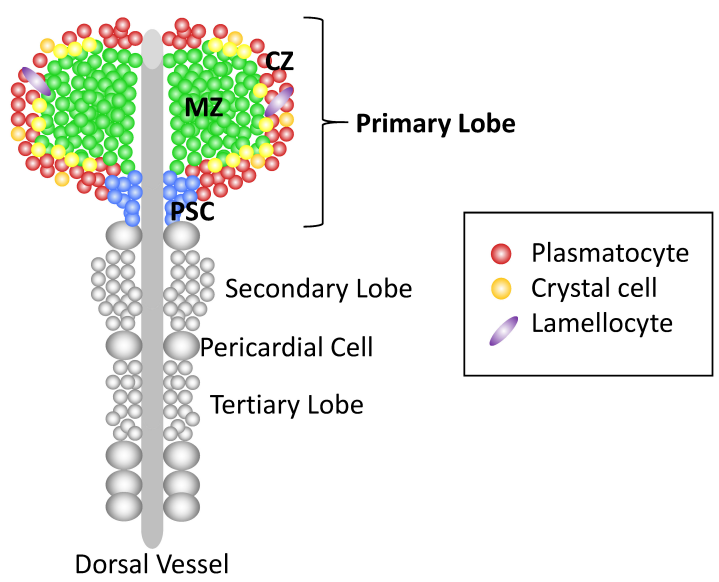

Fig. 1. Drosophila larval lymph gland. Blood cells that proliferate and differentiate during the larval stages reside in the primary lobe of the lymph gland. PSC (blue), MZ (green), CZ (red, orange, and purple - plasmatocytes, crystal cells, and lamellocytes, respectively). Yellow cells in between the $M Z$ and the $C Z$ are differentiating blood cells that exhibit characteristics of both progenitors and mature blood cells.

ISSN: 1976-670X (electronic edition)

Copyright (C) 2015 by the The Korean Society for Biochemistry and Molecular Biology

(c) This is an open-access article distributed under the terms of the Creative Commons Attribution Non-Commercial License (http://creativecommons.org/licenses/by-nc/3.0) which permits unrestricted non-commercial use, distribution, and reproduction in any medium, provided the original work is properly cited. 
topoiesis. Recently, an additional phase, larval hematopoiesis, has been recognized and found to play a role in the segmental colonization and expansion of larval circulating blood cells with the support of the peripheral nervous system. Larval hematopoiesis expands the pool of embryonic blood cells, enabling embryonic blood cells to persist and function properly during larval stages (13). During metamorphosis, blood cells in the lymph gland are dispersed into the circulation, and are in great need as they play major roles in cell engulfment, together with embryonic-origin blood cells (14). Thus, unless otherwise challenged, blood cells produced by the lymph gland do not participate in the larval blood circulation and only start to be released at the onset of pupariation (14).

\section{IMMUNE CHALLENGES AND THE BLOOD SYSTEM}

In the wild, flies commonly confront dangers of microbial infection, physical injury, and animal infestation as they feed in a microorganism-enriched environment. As a defense strategy, cellular and molecular mechanisms involving a multi-layered innate immunity are well developed in Drosophila, and are phylogenetically conserved in higher organisms (15). Drosophila can mount both cellular and humoral responses when attacked by bacteria, fungi, parasitic animals, and viruses (16). The humoral response is largely mediated by the expression of antimicrobial peptide (AMP) families, produced by tissues such as the fat body - equivalent to mammalian liver and adipose tissue - and released into the hemolymph to attack infectious microbes directly $(15,17,18)$. The Drosophila immune system recognizes various types of infectious microbes and specifically induces corresponding AMPs from specific cell types, under the control of the Toll or IMD pathway (19). In addition to the AMPs, oral infection induces reactive oxygen species (ROS) in the intestinal epithelia, suggesting that there are multiple layers of humoral immune responses upon microbial infection $(20,21)$.

The cellular immune response occurs simultaneously with the humoral response at the level of the blood cells. Plasmatocytes are responsible for the removal of small particles in the hemolymph, such as microorganisms and apoptotic cell debris (22). Receptors on the surface of plasmatocyte recognize target particles and modify the cytoskeleton to engulf, followed by a series of phagosome formation and disruption events. Several receptors involved in phagocytosis have been reported. A CD36 homolog, croquemort, is responsible for scavenging apoptotic cells in the embryo (23). In addition, members of the scavenger receptor family (dSR-Cl), the EGF-domain protein Eater, and the Ig-domain protein Dscam mediate bindings to Gram-negative and Gram-positive bacteria (24-27). Encapsulation is a powerful immune reaction against parasitic invaders carried out by a specialized blood cell, the lamellocyte. Lamellocytes only appear in the presence of invaders whose size is bigger than the phagocytic capacity of plasmatocytes (6). Active encapsulation has been analyzed using wasp in- festation, in which wasps lay their eggs into the larval hemocoel (28). Once the wasp egg is detected by plasmatocytes through an unidentified signal, massive differentiation of lamellocytes takes place, either by differentiation of existing circulating plasmatocytes (29) or differentiation of progenitors in the lymph gland (30). JAK/STAT, JNK, Toll pathways, chromatin remodeling, and the PSC-driven EGF signal have been identified as signals responsible for the differentiation and proliferation of lamellocytes $(11,31-34)$. Lamellocytes form a multilayered capsule around a parasitic particle, followed by subsequent melanization and particle breakdown. Another immune reaction that involves both humoral and cellular components of innate immunity is melanization, carried out by crystal cells. Melanization is controlled by a series of serine protease cascades that ultimately produce active prophenol oxidase, and this enzyme catalyzes tyrosine-derived phenols to form melanin. Several intermediate compounds generated during melanin synthesis are cytotoxic, so they participate in killing pathogens, indicating the humoral activity of melanization (35). The biggest reservoir of prophenol oxidase is the crystal cells. Mature crystal cells accumulate unidentified crystalline proteins, including prophenol oxidase, in the cytoplasm and are readily burst upon a specific signal, JNK, to release and activate the stored proteins (9). Thus, disruption of crystal cells is tightly associated with melanization at the level of the blood cells, which, in turn, leads to the humoral killing processes in a systematic manner.

\section{REACTIVE OXYGEN SPECIES AND THE BLOOD SYSTEM}

An interesting angle that has been identified with the Drosophila lymph gland is that wildtype Medullary zone cells contain significantly high ROS levels compared with their neighboring differentiated progeny (36). Scavenging ROS from the blood progenitors, by overexpression of antioxidants such as GTPx, retards their differentiation into mature blood cells. In contrast, increasing ROS above their normal levels by reducing Sod2 triggers precocious differentiation of mature blood cells. These studies thus provide the new insight that moderately high levels of ROS in blood progenitors serve as developmental signals and are essential for the maintenance of progenitors, while excessive ROS are harmful and deleterious to the cells. A recent study has found a detailed cellular mechanism underlying how high ROS expression in the progenitors is involved in both the maintenance and differentiation of blood cells at specific points in development (37). High ROS levels prime the blood progenitors in the early larval stages and are required for the expression of the adherent junction protein, E-cadherin, that is known to control stem cell integrity and facilitate signal transduction (38). Intriguingly, high levels of ROS are also detected in mammalian hematopoietic stem cells (HSCs) as well as in the common myeloid progenitors (CMPs), and maintaining moderate levels of ROS is key for their survival and normal cell cycle entry (39). 
In addition to the role of ROS in cell fate decisions, ROS are also involved in the early proliferation of blood progenitors in the lymph gland (40). Increases in Target of rapamycin (TOR) signaling in the lymph gland cause its overgrowth, along with the elevated levels of ROS. Additionally, a decrease in ROS levels in the high TOR activity background causes abnormal early proliferation of the lymph gland to return to the normal proliferation profile, indicating a role of ROS in proliferation. Consistent with this, a genetic modifier screen of the leukemic gene, AML1-ETO identified FoxO and superoxide dismutase 2 (SOD2) as suppressors of the leukemic phenotype via reduction of ROS (41). Overexpression of AML1-ETO in the larval blood results in the aberrant proliferation of circulating blood cells that can cause the formation of melanotic tumors. This phenotype is due to an increase in the circulating blood progenitors that express high levels of ROS, similar to the progenitors found in the lymph gland. Overexpression of FoxO or SOD2 in the circulating blood progenitors is sufficient to reduce the number of ROS-positive progenitors, indicating a role for ROS in the proliferation of blood progenitors. The role of high ROS has been studied extensively, particularly with regard to effects in apoptosis and the stress response. However, studies using the Drosophila blood system highlight the physiological roles of internal ROS in the cellular and developmental signaling that are essential for cell fate decisions as well as blood system integrity. Given that ROS levels are influenced by an animal's physiological status and by chemicals that alter redox status (37), it is possible that blood progenitors can be used as a redox sensor that monitors environmental toxicity.

\section{HYPOXIA AND THE BLOOD SYSTEM}

An adequate oxygen supply is one of the most critical determinants of an aerobic organism's survival. A representative hypoxic response mechanism functions mainly via hypoxia inducible factor HIF1, which recognizes physiological oxygen levels and transduces environmental information into gene expression (42). At the organismal level in Drosophila, tracheal cells respond to hypoxia with a heightened sensitivity to declining oxygen levels and undergo tracheal branch remodeling for the better reception of oxygen (43). In addition to the trachea, Drosophila blood cells are highly susceptible to hypoxic condition and increase the number of mature crystal cells in the lymph gland (10). Although the physiological role of these crystal cells in the hypoxic response is still unclear, it is possible that the crystal cells react to hypoxia in the way that the hemoglobin does in vertebrates or is preparation for possible injuries that may be caused by prolonged hypoxia. Interestingly, unlike other somatic cells, crystal cells stably express Sima, the Drosophila ortholog of Hifa, even under normoxic conditions. Accordingly, either increase in Sima due to hypoxic stress or disruption of developmental Sima by modifying the Sima-Notch interaction further expands the number of crystal cells in the lymph gland, indicating that Sima in the crystal cells plays dual roles in both the stress response and developmental control of cell fate decision. It is noteworthy that vertebrate myeloid cells also maintain high Hif1 $\alpha$ in normoxic conditions to maintain their cellular energy pools and the ability to mount an inflammatory response (44), and this conservation indicates a possible parallel mechanism between the Drosophila blood system and that of vertebrates.

A study with adult hemocytes provides an interesting aspect that overexpression of Hsp70 (Heat shock protein 70) in blood cells contributes to a remarkable survival benefit in severe hypoxia and oxidative stress by inhibition of systemic ROS (45). In addition to the systemic role of blood cells in immunity, this study provides another example of a systemic function of hemocytes in the stress response, which is not yet fully understood.

\section{NUTRITION AND THE BLOOD SYSTEM}

Nutritional deprivation directly impinges on the maintenance of blood progenitors in the lymph gland and induces differentiation of mature blood cells (40, 46-48). Indeed, 24 h starvation of third instar larvae induces precocious differentiation of mature blood cells and a concomitant decrease in the progenitors. Moreover, chronic starvation, $>48 \mathrm{~h}$, results in blood cell differentiation accompanied by a decrease in the size of the PSC and the lymph gland. These phenomena are related to the Drosophila insulin-like growth factor 2 (Ilp2), which is a reminiscent of the mammalian insulin (49) and plays a key role in nutrition-mediated tissue growth. Systemic insulin secreted from the brain neuroendocrine cells is perceived by the insulin receptor $(\operatorname{InR})$ either in the PSC or in the $M Z$ cells, and is required for proper maintenance of the lymph gland integrity. Lack of InR in the PSC causes a reduction in the niche size, which indirectly affects progenitor cell fate through changes in the expression of Hedgehog ( $\mathrm{Hh}$ (8). Moreover, loss of InR in MZ cells gives rise to precocious differentiation of mature blood cells through a cell-autonomous PI3K/AKT and TOR pathway that, in turn, controls Wingless in the $M Z$ cells $(40,46-48)$. These studies suggest that systemic nutrient and insulin signaling impact the local lymph glandbased signals to achieve blood homeostasis, and loss of the nutritional signal forces blood cells to differentiate. Interestingly, acute starvation generates hallmarks of inflammatory responses, including lamellocyte differentiation, crystal cell rupture, and infiltration of blood cells into the fat body, similar to the chronic inflammation phenotype observed in metabolic disorders (47). Moreover, it has been identified that there is a coordinated interaction between immune and metabolic signals to reallocate energy utilization from non-essential processes, such as growth, to more immediate needs, such as immunity (50). Thus, it is possible that starvation makes the blood progenitors withdraw their stemness and build up the blood cell repertoire to reinforce cellular immunity. From a developmental point of view, insulin-mediated blood regulation occurs naturally during the developmental timeline. Differenti- 
ation of the mature blood cells takes place in accordance with animal's feeding behavior, and the lymph gland finally disintegrates upon pupariation when blood cells are in great needs during metamorphosis (14). Insulin- and nutrition-mediated stem cell control mechanisms are conserved in other stem cell types in Drosophila, indicating that acquiring proper amount of nutrition is universally indispensable for stem cell control, although the specific targets and roles of insulin in each stem cell vary.

\section{ADENOSINE AND THE BLOOD SYSTEM}

Homeostasis in the lymph gland is achieved by several factors including local maintenance signals from the PSC, cell-autonomous control of the $\mathrm{MZ}$ cells, systemic signals from the brain, and the equilibrium signal from differentiating cells in the $\mathrm{CZ}$ cells (51). It has been established that the PSC expresses $\mathrm{Hh}$, which is the primary signal of $M Z$ progenitors' maintenance through the activation of Cubitus interruptus (Ci) (8). In addition to the Hh pathway, Pvf1 originating from the PSC is transported to the maturing blood cells in the cortical zone, where it binds to its receptor, Pvr, leading to production of Adgf-A via activation of STAT. Adgf-A is a secreted adenosine deaminase that functions to maintain low levels of adenosine in the progenitors. While adenosine is necessary for early progenitor proliferation, high levels of it lead to continuous progenitor proliferation and loss of their maintenance. Thus in this process, a balance between Adgf-A and adenosine plays a key role in achieving blood progenitor homeostasis and the presence of adenosine is likely to operate in a mechanism similar to the "quorum sensing" found in the prokaryotic colonization (52). Thus, a critical level of adenosine is decisive for the $M Z$ population. Given that adenosine is a key precursor of various metabolic processes, adenosine-derived developmental signals may be associated with stress responses, particularly mitochondrial or metabolic stresses. Adenosine-associated developmental regulation is another example of a mechanism involved in both development and stress responses, making the system very efficient and sensitive to systemic environmental changes.

\section{OLFACTION AND THE BLOOD SYSTEM}

Niche-dependent mechanisms of hematopoietic stem/progenitor cell maintenance have been studied extensively in both vertebrates and invertebrates (53). Mechanisms independent of niche-derived signals that operate in a more systemic manner but affect blood progenitors have recently started to emerge (54). In addition to nutrition-directed blood regulation, olfactory sensation impacts the blood progenitors in the lymph gland via secretion of physiological levels of $\gamma$-aminobutyric acid (GABA) into the blood stream (55). GABA is expressed in a subset of neuroendocrine cells, and the release of GABA is highly dependent on olfactory stimulation. Thus, olfactory dysfunction decreases GABA secretion from the neuroendocrine cells and reduces levels of GABA in the bloodstream. Blood progenitors in the lymph gland express the metabotropic $G A B A_{B}$ receptor that allows these cells to sense GABA in the circulation, leading to an increase in their cytosolic calcium concentration, essential for blood progenitor maintenance. Although downstream targets of high calcium in the progenitors are unclear, it is possible that developmental signals in the $M Z$ cells are able to use the calcium and cross-talk to each other. GABA is an evolutionarily conserved molecule. In plants, GABA serves as a stress signal as well as a metabolite (56), while GABA in vertebrates has been studied extensively with regard to its neurotransmitter function (57). Interestingly, there are related studies that GABA can be measured in the bloodstream of mammals, including humans (58), and the $G_{A B A}$ receptor is expressed in primary human HSCs (59), indicating a possible parallel mechanism conserved in higher organisms. It is interesting to note that olfaction can be associated with the developmental timing and stress response. Because olfactory receptors are dissociated upon pupariation, premature loss of olfactory receptors during larval stages is reminiscent of pupariation when extensive mature blood cells are to differentiate and disintegrate from the lymph gland.

\section{PERSPECTIVE}

The overall physiological status of an organism is critical for determining stem and progenitor cell fate. Given that purpose of harboring stem/progenitor cells is to provide a regenerative capacity, systemic controls of stem/progenitor cells are critical to ensure their cell fate determination as stem cells are tightly associated with rapid growth, immunity/injury, and other environmental challenges to meet the needs of an organism (60). Simultaneously, there is a developmental timeline that larval stem/progenitors should follow to complete normal development. Thus, instead of using two different mechanisms for development and stress response, animals use identical mechanisms that allow easy conversion, depending on their external circumstances.

In the lymph gland, mature blood cells appear in the mid-to-late second instar larval life, followed by rapid differentiation in late third instar (6). At the onset of pupariation, the lymph gland disintegrates and bursts open to release blood cells (14). Even with no infection, mature blood cells are essential during metamorphosis to scavenge and phagocytose larval cells and bacteria released from the reforming gut. We can view the blood progenitor control mechanisms introduced in this review as signals that exist physiologically during larval development and, as the larvae approach pupariation, these processes are attenuated. As third instar larvae start wandering, the larval insulin level drops naturally (61). At this time, larvae generate a cuticle, causing reduced oxygen diffusion, and dissociate larval olfactory receptors to decrease sensory perception. Finally, remodeling of the gut releases chemical/ bacterial substances and metabolic intermediates, which are 
likely to induce immune responses, cause ROS induction, and change adenosine levels. Thus, all the conditions that facilitate blood differentiation are also developmental consequences of the initiation of pupariation. Interestingly, different types of stresses are also sensed by these pathways and cause precocious progenitor differentiation, thus functioning as stress sensors in the system.

It seems likely that increased differentiation of mature blood cells in various stress responses may facilitate the initiation of more rapid immune reactions by acquiring additional challenges. However, whether this blood differentiation response is directly related to the systems' protective mechanism is not yet clear. It will be interesting to understand this mechanism, of which process contains primitive features of boosted immunity by the increased blood repertoire, similar to the adaptive immune system in vertebrates. Given that larvae are challenged by various external changes, it is possible that Drosophila has additional and novel mechanisms that can affect both development and stress responses.

\section{ACKNOWLEDGEMENTS}

I thank T. Mukherjee for helpful comments and discussions. This work was supported by Basic Science Research Program through the National Research Foundation of Korea (NRF) funded by the Ministry of Science, ICT \& Future Planning (NRF-2014R1A1A1002685) and the research fund of Hanyang University (HY-2014-N).

\section{REFERENCES}

1. Makhijani K and Brückner K (2012) Of blood cells and the nervous system: hematopoiesis in the Drosophila larva. Fly 6, 254-260

2. Tepass U, Fessler LI, Aziz A and Hartenstein V (1994) Embryonic origin of hemocytes and their relationship to cell death in Drosophila. Development 120, 1829-1837

3. Wood W and Jacinto A (2007) Drosophila melanogaster embryonic haemocytes: masters of multitasking. Nat Rev Mol Cell Biol 8, 542-551

4. Evans CJ, Hartenstein V and Banerjee U (2003) Thicker than blood: conserved mechanisms in Drosophila and vertebrate hematopoiesis. Dev Cell 5, 673-690

5. Lanot R, Zachary D, Holder F and Meister M (2001) Postembryonic hematopoiesis in Drosophila. Dev Biol 230, 243-257

6. Jung SH, Evans CJ, Uemura C and Banerjee U (2005) The Drosophila lymph gland as a developmental model of hematopoiesis. Development 132, 2521-2533

7. Krzemień J, Dubois L, Makki R, Meister M, Vincent A and Crozatier M (2007) Control of blood cell homeostasis in Drosophila larvae by the posterior signalling centre. Nature 446, 325-328

8. Mandal L, Martinez-Agosto JA, Evans CJ, Hartenstein V and Banerjee U (2007) A Hedgehog- and Antennapediadependent niche maintains Drosophila haematopoietic precursors. Nature 446, 320-324

9. Lebestky T, Chang $T$, Hartenstein $V$ and Banerjee $U$ (2000) Specification of Drosophila hematopoietic lineage by conserved transcription factors. Science 288, 146-149

10. Mukherjee T, Kim WS, Mandal L and Banerjee U (2011) Interaction between Notch and Hif-alpha in development and survival of Drosophila blood cells. Science 332, 1210-1213

11. Sinenko SA, Shim J and Banerjee U (2012) Oxidative stress in the haematopoietic niche regulates the cellular immune response in Drosophila. EMBO Rep 13, 83-89

12. Rizki TM and Rizki RM (1992) Lamellocyte differentiation in Drosophila larvae parasitized by Leptopilina. Dev Comp Immunol 16, 103-110

13. Makhijani K, Alexander B, Tanaka T, Rulifson E and Brückner K (2011) The peripheral nervous system supports blood cell homing and survival in the Drosophila larva. Development 138, 5379-5391

14. Grigorian M, Mandal L and Hartenstein V (2011) Hematopoiesis at the onset of metamorphosis: terminal differentiation and dissociation of the Drosophila lymph gland. Dev Genes Evol 221, 121-131

15. Lemaitre B and Hoffmann J (2007) The host defense of Drosophila melanogaster. Annu Rev Immunol 25, 697-743

16. Ligoxygakis $P$ (2013) Genetics of immune recognition and response in Drosophila host defense. Adv Genet 83, 71-97

17. Imler JL and Bulet P (2005) Antimicrobial peptides in Drosophila: structures, activities and gene regulation. Chem Immunol Allergy 86, 1-21

18. Wicker C, Reichhart JM, Hoffmann D, Hultmark D, Samakovlis C and Hoffmann JA (1990) Insect immunity. Characterization of a Drosophila cDNA encoding a novel member of the diptericin family of immune peptides. J Biol Chem 265, 22493-22498

19. Ferrandon D, Imler JL, Hetru C and Hoffmann JA (2007) The Drosophila systemic immune response: sensing and signalling during bacterial and fungal infections. Nat Rev Immunol 7, 862-874

20. Ha EM, Oh CT, Bae YS and Lee WJ (2005) A direct role for dual oxidase in Drosophila gut immunity. Science 310, 847-850

21. Ha EM, Oh CT, Ryu JH et al (2005) An antioxidant system required for host protection against gut infection in Drosophila. Dev Cell 8, 125-132

22. Meister M (2004) Blood cells of Drosophila: cell lineages and role in host defence. Curr Opin Immunol 16, 10-15

23. Franc NC, Heitzler P, Ezekowitz RA and White K (1999) Requirement for croquemort in phagocytosis of apoptotic cells in Drosophila. Science 284, 1991-1994

24. Rämet M, Pearson A, Manfruelli P et al (2001) Drosophila scavenger receptor $\mathrm{Cl}$ is a pattern recognition receptor for bacteria. Immunity 15, 1027-1038

25. Pearson A, Lux A and Krieger M (1995) Expression cloning of dSR-Cl, a class $\mathrm{C}$ macrophage-specific scavenger receptor from Drosophila melanogaster. Proc Natl Acad Sci U S A 92, 4056-4060

26. Kocks C, Cho JH, Nehme N et al (2005) Eater, a transmembrane protein mediating phagocytosis of bacterial pathogens in Drosophila. Cell 123, 335-346 
27. Watson FL, Püttmann-Holgado R, Thomas $F$ et al (2005) Extensive diversity of Ig-superfamily proteins in the immune system of insects. Science 309, 1874-1878

28. Russo J, Dupas S, Frey F, Carton Y and Brehelin M (1996) Insect immunity: early events in the encapsulation process of parasitoid (Leptopilina boulardi) eggs in resistant and susceptible strains of Drosophila. Parasitology 112 ( Pt 1), 135-142

29. Stofanko M, Kwon SY and Badenhorst P (2010) Lineage tracing of lamellocytes demonstrates Drosophila macrophage plasticity. PLoS One 5, e14051

30. Krzemien J, Oyallon J, Crozatier M and Vincent A (2010) Hematopoietic progenitors and hemocyte lineages in the Drosophila lymph gland. Dev Biol 346, 310-319

31. Luo H, Hanratty WP and Dearolf CR (1995) An amino acid substitution in the Drosophila hopTum-I Jak kinase causes leukemia-like hematopoietic defects. EMBO J 14, $1412-1420$

32. Qiu P, Pan PC and Govind S (1998) A role for the Drosophila Toll/Cactus pathway in larval hematopoiesis. Development 125, 1909-1920

33. Zettervall CJ, Anderl I, Williams MJ et al (2004) A directed screen for genes involved in Drosophila blood cell activation. Proc Natl Acad Sci U S A 101, 14192-14197

34. Kwon SY, Xiao H, Glover BP, Tjian R, Wu C and Badenhorst P (2008) The nucleosome remodeling factor (NURF) regulates genes involved in Drosophila innate immunity. Dev Biol 316, 538-547

35. Söderhäll K and Cerenius L (1998) Role of the prophenoloxidase-activating system in invertebrate immunity. Curr Opin Immunol 10, 23-28

36. Owusu-Ansah E and Banerjee U (2009) Reactive oxygen species prime Drosophila haematopoietic progenitors for differentiation. Nature 461, 537-541

37. Gao H, Wu X, Simon L and Fossett N (2014) Antioxidants maintain e-cadherin levels to limit Drosophila prohemocyte differentiation. PloS One 9, e107768

38. Harris TJ and Tepass U (2010) Adherens junctions: from molecules to morphogenesis. Nat Rev Mol Cell Biol 11, 502-514

39. Tothova Z and Gilliland DG (2007) FoxO transcription factors and stem cell homeostasis: insights from the hematopoietic system. Cell Stem Cell 1, 140-152

40. Dragojlovic-Munther M and Martinez-Agosto JA (2012) Multifaceted roles of PTEN and TSC orchestrate growth and differentiation of Drosophila blood progenitors. Development 139, 3752-3763

41. Sinenko SA, Hung T, Moroz T et al (2010) Genetic manipulation of AML1-ETO-induced expansion of hematopoietic precursors in a Drosophila model. Blood 116, 4612-4620

42. Gorr TA, Gassmann M and Wappner P (2006) Sensing and responding to hypoxia via HIF in model invertebrates. J Insect Physiol 52, 349-364

43. Jarecki J, Johnson E and Krasnow MA (1999) Oxygen regulation of airway branching in Drosophila is mediated by branchless FGF. Cell 99, 211-220

44. Cramer T, Yamanishi Y, Clausen BE et al (2003) HIF-1alpha is essential for myeloid cell-mediated inflammation. Cell 112, 645-657

45. Azad P, Ryu J and Haddad GG (2011) Distinct role of Hsp70 in Drosophila hemocytes during severe hypoxia. Free Radic Biol Med 51, 530-538

46. Benmimoun B, Polesello C, Waltzer $L$ and Haenlin $M$ (2012) Dual role for Insulin/TOR signaling in the control of hematopoietic progenitor maintenance in Drosophila. Development 139, 1713-1717

47. Shim J, Mukherjee T and Banerjee U (2012) Direct sensing of systemic and nutritional signals by haematopoietic progenitors in Drosophila. Nat Cell Biol 14, 394-400

48. Tokusumi Y, Tokusumi T, Shoue DA and Schulz RA (2012) Gene regulatory networks controlling hematopoietic progenitor niche cell production and differentiation in the Drosophila lymph gland. PloS One 7, e41604

49. Hietakangas $\vee$ and Cohen SM (2009) Regulation of tissue growth through nutrient sensing. Annu Rev Genet 43, $389-410$

50. DiAngelo JR, Bland ML, Bambina S, Cherry $S$ and Birnbaum MJ (2009) The immune response attenuates growth and nutrient storage in Drosophila by reducing insulin signaling. Proc Natl Acad Sci U S A 106, 20853-20858

51. Mondal BC, Mukherjee T, Mandal L et al (2011) Interaction between differentiating cell- and niche-derived signals in hematopoietic progenitor maintenance. Cell 147, 1589-1600

52. Ng WL and Bassler BL (2009) Bacterial quorum-sensing network architectures. Annu Rev Genet 43, 197-222

53. Mercier FE, Ragu C and Scadden DT (2012) The bone marrow at the crossroads of blood and immunity. Nat Rev Immunol 12, 49-60

54. Gancz D and Gilboa L (2013) Insulin and Target of rapamycin signaling orchestrate the development of ovarian niche-stem cell units in Drosophila. Development 140, 4145-4154

55. Shim J, Mukherjee T, Mondal BC et al (2013) Olfactory control of blood progenitor maintenance. Cell 155, 1141-1153

56. Roberts MR (2007) Does GABA Act as a Signal in Plants?: Hints from Molecular Studies. Plant Signal Behav 2, 408-409

57. Li K and Xu E (2008) The role and the mechanism of gamma-aminobutyric acid during central nervous system development. Neurosci Bull 24, 195-200

58. Ferkany JW, Smith LA, Seifert WE, Caprioli RM and Enna SJ (1978) Measurement of gamma-aminobutyric acid (GABA) in blood. Life Sci 22, 2121-2128

59. Steidl U, Bork S, Schaub S et al (2004) Primary human $\mathrm{CD} 34+$ hematopoietic stem and progenitor cells express functionally active receptors of neuromediators. Blood 104, 81-88

60. Shim J, Gururaja-Rao S and Banerjee U (2013) Nutritional regulation of stem and progenitor cells in Drosophila. Development 140, 4647-4656

61. Slaidina M, Delanoue R, Gronke S, Partridge L and Léopold P (2009) A Drosophila insulin-like peptide promotes growth during nonfeeding states. Dev Cell 17, 874-884 